\title{
ON TOTAL REALITY OF MEROMORPHIC FUNCTIONS
}

\author{
ALEX DEGTYAREV, TORSTEN EKEDAHL, ILIA ITENBERG, BORIS SHAPIRO, \\ AND MICHAEL SHAPIRO
}

\begin{abstract}
We show that if a meromorphic function of degree at most four on a real algebraic curve of an arbitrary genus has only real critical points then it is conjugate to a real meromorphic function after a suitable projective automorphism of the image.
\end{abstract}

\section{INTRODUCTION}

Let $\gamma: \mathbb{C P}^{1} \rightarrow \mathbb{C P}^{n}$ be a rational curve in $\mathbb{C P}^{n}$. We say that a point $t \in \mathbb{C P}^{1}$ is a flattening point of $\gamma$ if the osculating frame formed by $\gamma^{\prime}(t), \gamma^{\prime \prime}(t), \ldots, \gamma^{(n)}(t)$ is degenerate. In other words, flattening points of $\gamma(t)=\left(\gamma_{0}(t): \gamma_{2}(t): \cdots: \gamma_{n}(t)\right)$ are roots of the Wronskian

$$
W\left(\gamma_{0}, \ldots, \gamma_{n}\right)=\left|\begin{array}{ccc}
\gamma_{0} & \ldots & \gamma_{n} \\
\gamma_{0}^{\prime} & \ldots & \gamma_{n}^{\prime} \\
& \ldots & \\
\gamma_{0}^{(n)} & \ldots & \gamma_{n}^{(n)}
\end{array}\right| .
$$

In 1993 B. and M. Shapiro made the following claim which we will refer to as rational total reality conjecture.

Conjecture 1.1. If all flattening points of a rational curve $\mathbb{C P}^{1} \rightarrow \mathbb{C P}^{n}$ lie on the real line $\mathbb{R} P^{1} \subset \mathbb{C P}^{1}$ then the curve is conjugate to a real algebraic curve under an appropriate projective automorphism of $\mathbb{C P}^{n}$.

Notice that coordinates $\gamma_{i}$ of the rational curve $\gamma$ are homogeneous polynomials of a certain degree, say $d$. Considering them as vectors in the space of homogeneous degree $d$ polynomials we can reformulate the above conjecture as a statement of total reality in Schubert calculus, see [7, 11- 15], 16]. Namely, for any $0 \leq d<n$ let $t_{1}<t_{2}<\cdots<t_{(n+1)(d-n)}$ be a sequence of real numbers and $\mathbf{r}: \mathbb{C} \rightarrow \mathbb{C}^{\mathbf{d}+\mathbf{1}}$ be a rational normal curve with coordinates $r_{i}(t)=t^{i}, i=\overline{0, d}$. Denote by $T_{i}$ the osculating $(d-n)$-dimensional plane to $\mathbf{r}$ at the moment $t=t_{i}$. Then the above rational total reality conjecture is equivalent to the following claim.

Conjecture 1.2 (Schubert calculus interpretation). In the above notation any $(n+1)$-dimensional subspace in $\mathbb{C}^{d+1}$ which meets all $(n+1)(d-n)$ subspaces $T_{i}$ nontrivially is real.

2000 Mathematics Subject Classification. Primary 14P05; Secondary 14P25.

I.I. is partially supported by the ANR-network "Interactions et aspects énumératifs des géométries réelle, tropicale et symplectique".

M.S. is partially supported by NSF grants DMS-0401178, by the BSF grant 2002375 and by the Institute of Quantum Science, MSU. 
It was first supported by extensive numerical evidences, see [11- [15, 16] and later settled for $n=1$, see 3 . The case $n \geq 2$ resisted all efforts for a long time. In fall 2005 the authors were informed by A. Eremenko and A. Gabrielov that they were able to prove Conjecture 1.1 for plane rational quintics. Just few months later it was completely established by E. Mukhin, V. Tarasov, and A. Varchenko in 8].

Their proof reveals the deep connection between Schubert calculus and theory of integrable system and is based on the Bethe ansatz method in the Gaudin model. More exactly, conjectures 1 and 2 are reduced to the question of reality of $(n+$ 1)-dimensional subspaces of the space $V$ of polynomials of degree $d$ with given asymptotics at infinity and fixed Wronskian. Choosing a base in such a subspace we get the rational curve $\mathbb{C P}^{1} \rightarrow \mathbb{C P}^{n}$, whose flattening points coincide with the roots of the above mentioned Wronskian. The subspaces with desired properties are constructed explicitly using properties of spectra of Gaudin Hamiltonians. Namely, relaxing the reality condition these polynomial subspaces are labeled by common eigenvectors of Gaudin Hamiltonians, one-parameter families of commuting linear maps on some vector space, $H_{1}(x), \ldots, H_{n+1}(x): V \rightarrow V$. The subspace, labeled by an eigenvector, is the kernel of a certain linear differentail operator of order $n+1$, assigned to each eigenvector of the Hamiltonians. The coefficents of that differentail operator, are the eigenvalues of the Hamiltonians on that eigenvector. It turns out that in the case of real rooted Wronskians Gaudin Hamiltonians are symmetric with respect to the so-called tensor Shapovalov form, and thus have real spectra. Moreover, their eigenvalues are real rational functions. This fact implies that the kernels of the above fundamental differential operators are real subspaces in $V$ which concludes the proof.

Meanwhile two different generalizations of the original conjectures (both dealing with the case $n=1$ ) were suggested in [4] and [5]. The former replaces the condition of reality of critical points by the existence of separated collections of real points such that a meromorphic function takes the same value on each set. The latter discusses the generalization of the total reality conjecture to higher genus curves.

The present paper is the sequel of $[\underline{5}$. Here we prove the higher genus version of the total reality conjecture for all meromorphic funtions of degree at most four.

For reader's convenience and to make the paper self-contained we included some of results of [5] here. We start with some standard notation.

Definition. A pair $(\mathcal{C}, \sigma)$ consisting of a compact Riemann surface $\mathcal{C}$ and its antiholomorphic involution $\sigma$ is called a real algebraic curve. The set $\mathcal{C}_{\sigma} \subset \mathcal{C}$ of all fixed points of $\sigma$ is called the real part of $(\mathcal{C}, \sigma)$.

If $(\mathcal{C}, \sigma)$ and $(\mathcal{D}, \tau)$ are real curves (varieties) and $f: \mathcal{C} \rightarrow \mathcal{D}$ a holomorphic map, then we denote by $\bar{f}$ the holomorphic map $\tau \circ f \circ \sigma$. Notice that $f$ is real if and only if $\bar{f}=f$.

The main question we discuss below is as follows.

Main Problem. Given a meromorphic function $f:(\mathcal{C}, \sigma) \rightarrow \mathbb{C P}^{1}$ such that i) all its critical points and values are distinct;

ii) all its critical points belong to $\mathcal{C}_{\sigma}$;

is it true that that $f$ becomes a real meromorphic function after an appropriate choice of a real structure on $\mathbb{C P}^{1}$ ?

Definition. We say that the space of meromorphic functions of degree $d$ on a genus $g$ real algebraic curve $(\mathcal{C}, \sigma)$ has the total reality property (or is totally real) if the 
Main Problem has the affirmative answer for any meromorphic function from this space which satisfies the above assumptions. We say that a pair of positive integers $(g, d)$ has a total reality property if the space of meromorphic functions of degree $d$ is totally real on any real algebraic curve of genus $g$.

The following results were proven in [5] (see Theorem 1 and Corollary 1 there).

Theorem 1.3. The space of meromorphic functions of any degree $d$ which is a prime on any real curve $(\mathcal{C}, \sigma)$ of genus $g$ which additionally satisfies the inequality: $g>\frac{d^{2}-4 d+3}{3}$ has the total reality property.

Corollary 1.4. The total reality property holds for all meromorphic functions of degrees 2,3 , i.e. for all pairs $(g, 2)$ and $(g, 3)$.

The proof of the Theorem 1.3 is based on the following observation. Consider the space $\mathbb{C P}^{1} \times \mathbb{C P}^{1}$ equipped with the involution $s:(x, y) \mapsto(\bar{y}, \bar{x})$ which we call the involutive real structure (here $\bar{x}$ and $\bar{y}$ stand for the complex conjugates of $x$ and $y$ with respect to the standard real structure in $\left.\mathbb{C P}^{1}\right)$. The pair $\mathbf{E l l}=\left(\mathbb{C P}^{1} \times \mathbb{C P}^{1}, \mathbf{s}\right)$ is usually referred to as the standard ellipsoid, see [6]. (Sometimes by the ellipsoid one means the set of fixed points of $s$ on $\left.\mathbb{C P}^{1} \times \mathbb{C P}^{1}\right)$. The next statement translates the problem of total reality into the question of (non)existence of certain real algebraic curves on Ell.

Proposition 1.5. For any positive integer $g$ and prime $d$ the total reality property holds for the pair $(g, d)$ if and only if there is no real algebraic curve on Ell with the following properties:

i) its geometric genus equals $\mathrm{g}$;

ii) its bi-degree as a curve on $\mathbb{C P}^{1} \times \mathbb{C P}^{1}$ equals $(d, d)$;

iii) its only singularities are $2 d-2+2 g$ real cusps on Ell and possibly some number of (not necessarily transversal) intersections of smooth branches.

Extending slightly the arguments proving Proposition 1.5 one gets the following statement.

Proposition 1.6. The total reality property holds for all real meromorphic functions, i.e. for all pairs $\left(g^{\prime}, d^{\prime}\right)$ if and only if for no pair $(g, d), d>1$ there exists a real algebraic curve on Ell satisfying conditions i) - iii) of Proposition 1.5.

The main result of the present paper obtained using a version of Proposition 1.5 and technique related to integer lattices and $K 3$-surfaces is as follows.

Theorem 1.7. The total reality property holds for all meromorphic functions of degree 4 , i.e. for all pairs $(g, 4)$.

The structure of the note is as follows. $\S 2$ contains the proofs of Theorem 1.3 Corollary 1.4 and reduction of Theorem [1.7 to the question of nonexistence of a real curve $\mathcal{D}$ on Ell of bi-degree $(4,4)$ with eight real cusps and no other singularities. $\S 3$ contains a proof of nonexistence of such curve $\mathcal{D}$, while $\S$ 迎 contains a number of remarks and open problems.

Acknowledgements. The authors are grateful to A. Gabrielov, A. Eremenko, R. Kulkarni, B. Osserman, V. Tarasov, A. Vainshtein, and A. Varchenko for discussions of the topic. The third, fourth and fifth authors want to acknowledge the hospitality of MSRI in Spring 2004 during the program 'Topological methods in real algebraic geometry' which gave them a large number of valuable research inputs. 


\section{Proofs}

If not mentioned explicitly we assume below that $\mathbb{C P}^{1}$ is provided with its standard real structure. Real meromorphic functions on a real algebraic curve $(\mathcal{C}, \sigma)$ can be characterized in the following way.

Proposition 2.1. If $(\mathcal{C}, \sigma)$ is a proper irreducible real curve and $f: \mathcal{C} \rightarrow \mathbb{C P}^{1}$ is a non-constant holomorphic map, then $f$ is real for some real structure on $\mathbb{C P}^{1}$ if and only if there is a Möbius transformation $\varphi: \mathbb{C P}^{1} \rightarrow \mathbb{C P}^{1}$ such that $\bar{f}=\varphi \circ f$.

Proof. Any real structure on $\mathbb{C P}^{1}$ is of the form $\tau \circ \phi$ for a complex Möbius transformation $\phi$ and $\tau$ the standard real structure with $\bar{\phi} \circ \phi=$ id and conversely any such $\phi$ gives a real structure. If $f$ is real for such a structure we have $f=\tau \circ \phi \circ f \circ \sigma$, i.e., $\bar{f}=\varphi \circ f$ for $\varphi=\tau \circ \phi^{-1} \circ \tau$. Conversely, if $\bar{f}=\varphi \circ f$, then $f=\overline{\bar{f}}=\overline{\varphi \circ f}=\bar{\varphi} \circ \bar{f}=\bar{\varphi} \circ \varphi \circ f$ and as $f$ is surjective we get $\bar{\varphi} \circ \varphi=$ id. That means that $\phi:=\tau \circ \varphi^{-1} \circ \tau$, then $\phi$ defines a real structure on $\mathbb{C P}^{1}$ and by construction $f$ is real for that structure and the fixed one on $\mathcal{C}$.

Up to a real isomorphism there are only two real structures on $\mathbb{C P}^{1}$, the standard one and the one on an isotropic real quadric in $\mathbb{C P}^{2}$. The latter is distinguished from the former by not having any real points.

Assume now that $(\mathcal{C}, \sigma)$ is a proper irreducible real curve and $f: \mathcal{C} \rightarrow \mathbb{C P}^{1}$ a non-constant meromorphic function. It defines the holomorphic map

$$
\mathcal{C} \stackrel{(f, \bar{f})}{\longrightarrow} \mathbb{C P}^{1} \times \mathbb{C P}^{1}
$$

and if $\mathbb{C P}^{1} \times \mathbb{C P}^{1}$ is given the involutive real structure $s:(x, y) \rightarrow(\bar{y}, \bar{x})$ then it is clearly a real map. Now we can formulate the central technical result of this section.

\section{Proposition 2.2.}

(1) The image $\mathcal{D}$ of the curve $\mathcal{C}$ under the map $(f, \bar{f})$ is of type $(\delta, \delta)$ for some positive integer $\delta$ and if $\partial$ is the degree of the map $\mathcal{C} \rightarrow \mathcal{D}$ we have that $d=\delta \partial$, where $d$ is the degree of the original $f$.

(2) The function $f$ is real for some real structure on $\mathbb{C P}^{1}$ precisely when $\delta=1$.

(3) Assume that $\mathcal{C}$ is smooth and all the critical points of $f$ are real. Then all the critical points of $\psi: \widetilde{\mathcal{D}} \rightarrow \mathbb{C P}^{1}$, the composite of the normalization map $\widetilde{\mathcal{D}} \rightarrow \mathcal{D}$ and the restriction of the projection of $\mathbb{C P}^{1} \times \mathbb{C P}^{1}$ has all its critical points real.

Proof. The image of $\mathcal{C}$ under the real holomorphic map $(f, \bar{f})$ is a real curve so that $\mathcal{D}$ is a real curve in $\mathbb{C P}^{1} \times \mathbb{C P}^{1}$ with respect to its involutive real structure, i.e. a real curve on the ellipsoid Ell. Any such curve is of type $(\delta, \delta)$ for some positive integer $\delta$ since the involutive real structure permutes the two degrees. The rest of (1) follows by using the multiplicativity of degrees for the maps $f: \mathcal{C} \rightarrow \mathcal{D} \rightarrow \mathbb{C P}^{1}$, where the last map is projection on the first factor.

As for (2) assume first that $f$ can be made real for some real structure on $\mathbb{C P}^{1}$. By Proposition 2.1 there is a Möbius transformation $\varphi$ such that $\bar{f}=\varphi \circ f$ but that in turn means that $(f, \bar{f})$ maps $\mathcal{C}$ into the graph of $\varphi$ in $\mathbb{C P}^{1} \times \mathbb{C P}^{1}$. This graph is hence equal to $\mathcal{D}$ and is thus of type $(1,1)$. Conversely, assume that $\mathcal{D}$ is of type $(1,1)$. Then it is a graph of an isomorphism $\varphi$ from $\mathbb{C P}^{1}$ to $\mathbb{C P}^{1}$ and by construction $\bar{f}=\varphi \circ f$ so we conclude by another application of Proposition 2.1 
Finally, for (3) we have that the map $\mathcal{C} \rightarrow \mathcal{D}$ factors as a, necessarily real, map $h: \mathcal{C} \rightarrow \widetilde{\mathcal{D}}$ and then $f=\psi \circ h$. If $p t \in \widetilde{\mathcal{D}}$ is a critical point, then all points of $h^{-1}(p t)$ are critical for $f$ and hence by assumption real. As $h$ is real this implies that $p t$ is also real.

Part (2) of the above Proposition gives another reformulation of the total reality property for meromorphic functions.

Corollary 2.3. If a meromorphic function $f:(\mathcal{C}, \sigma) \rightarrow \mathbb{C P}^{1}$ of degree $d$ is real for some real structure on $\mathbb{C P}^{1}$ then the map $\mathcal{C} \stackrel{(f, \bar{f})}{\longrightarrow} \mathcal{D} \subset \mathbb{C P}^{1} \times \mathbb{C P}^{1}$ must have degree $d$ as well.

Remark 2.4. Notice that without the requirement of reality of $f$ the degree of $\mathcal{C} \stackrel{(f, \bar{f})}{\longrightarrow} \mathcal{D}$ can be any factor of $d$.

By a cusp we mean a curve singularity of multiplicity 2 and whose tangent cone is a double line. It has the local form $y^{2}=x^{k}$ for some integer $k \geq 3$ where $k$ is an invariant which we shall call its type. A cusp of type $k$ gives a contribution of $\lceil(k-1) / 2\rceil$ to the arithmetic genus of a curve. A cusp of type 3 will be called ordinary.

If $\mathcal{C}$ is a curve and $p_{1}, \ldots, p_{k}$ are its smooth points then consider the finite map $\pi: \mathcal{C} \rightarrow \mathcal{C}\left(p_{1}, \ldots, p_{k}\right)$ which is a homeomorphism and for which $\mathcal{O}_{\mathcal{C}\left(p_{1}, \ldots, p_{k}\right)} \rightarrow$ $\pi_{*} \mathcal{O}_{\mathcal{C}}$ is an isomorphism outside of $\left\{p_{1}, \ldots, p_{k}\right\}$ such that the image of the map $\mathcal{O}_{\mathcal{C}\left(p_{1}, \ldots, p_{k}\right), \pi\left(p_{i}\right)} \rightarrow \mathcal{O}_{\mathcal{C}, p_{i}}$ is the inverse image of $\mathbb{C}$ in $\mathcal{O}_{\mathcal{C}, p_{i}} / \mathfrak{m}_{p_{i}}^{2}$. In other words, $\mathcal{C}\left(p_{1}, \ldots, p_{k}\right)$ has ordinary cusps at all points $\pi\left(p_{i}\right)$.

Then $\pi$ has the following two (obvious) properties:

\section{Lemma 2.5.}

(1) A holomorphic map $f: \mathcal{C} \rightarrow X$ which is not an immersion at all the points $p_{1}, \ldots, p_{k}$ factors through $\pi$.

(2) If $\mathcal{C}$ is proper, then the arithmetic genus of $\mathcal{C}\left(p_{1}, \ldots, p_{k}\right)$ is $k$ plus the arithmetic genus of $\mathcal{C}$.

Proposition 2.6. Assume that $(\mathcal{C}, \sigma)$ is a smooth and proper real curve and let $f: \mathcal{C} \rightarrow \mathbb{C P}^{1}$ be a meromorphic function of degree $d$. If there are $k$ real points $p_{1}, \ldots, p_{k}$ on $\mathcal{C}$ which are critical points for $f$ and if $(f, \bar{f})$ gives a map of degree 1 from $\mathcal{C}$ to its image $\mathcal{D}$ in $\mathbb{C P}^{1} \times \mathbb{C P}^{1}$, then $g(\mathcal{C})+k \leq(d-1)^{2}$. If $g(\mathcal{C})+k=(d-1)^{2}$, then the map $h: \mathcal{C} \rightarrow \mathcal{D}$ factors to give an isomorphism $\mathcal{C}\left(p_{1}, \ldots, p_{k}\right) \stackrel{\sim}{\rightarrow} D$.

Proof. As $p_{i}$ is a real critical point it is a critical for $\bar{f}$ as well and hence for $(f, \bar{f})$. This implies by the first property for $\pi: \mathcal{C} \rightarrow \mathcal{C}\left(p_{1}, \ldots, p_{k}\right)$ that the map $\mathcal{C} \rightarrow D$ factors as $\mathcal{C} \rightarrow \mathcal{C}\left(p_{1}, \ldots, p_{k}\right) \rightarrow \mathcal{D}$ and hence the arithmetic genus of $\mathcal{C}\left(p_{1}, \ldots, p_{k}\right)$, which is $g(\mathcal{C})+k$ by the second property of $\mathcal{C}\left(p_{1}, \ldots, p_{k}\right)$, is less than or equal to the arithmetic genus of $\mathcal{D}$, which by the adjunction formula is equal to $(d-1)^{2}$. If we have equality then their genera are equal and hence the map $\mathcal{C}\left(p_{1}, \ldots, p_{k}\right) \rightarrow \mathcal{D}$ is an isomorphism.

Now it is easy to derive Proposition 1.5 from Proposition 2.2 Indeed, if a meromorphic function $f: \mathcal{C} \rightarrow \mathbb{C P}^{1}$ of a prime degree $d$ with all $2 g+2 d-2$ real critical points can not be made real then its image under $(f, \bar{f})$ in $\mathbb{C P}^{1} \times \mathbb{C P}^{1}$ is the real curve on Ell with $2 g+2 d-2$ real cusps and no other singularities different from 
intersections of smooth branches. (Intersections of smooth branches in the image might occur and are moreover necessary to produce the required genus.) Vice versa, assume that such a curve $\mathcal{D} \subset \mathbb{C P}^{1} \times \mathbb{C P}^{1}$ which is real in the involutive structure does exist. Let $\widetilde{\mathcal{D}}$ be the normalization of $\mathcal{D}$, and consider the natural birational projection map $\mu: \widetilde{\mathcal{D}} \rightarrow \mathcal{D}$. Define $f: \widetilde{\mathcal{D}} \rightarrow \mathbb{C P}^{1}$ as a composition $\widetilde{D} \rightarrow \mathcal{D} \rightarrow \mathbb{C P}^{1}$, where the last map is induced by the projection of $\mathbb{C P}^{1} \times \mathbb{C P}^{1}$ on the first factor. It remains to notice that all $2 g+2 d-2$ critical points of $f$ are real while $f$ can not be made real by Proposition 2.2

Similar arguments show the validity of Proposition 1.6 Indeed, assume that there exists a meromorphic function $\phi$ of some degree $d^{\prime}$ on a real curve $\mathcal{C}^{\prime}$ of some genus $g^{\prime}$ violating the total reality conjecture. Let $\mathcal{D}^{\prime} \subset \mathbb{C P}^{1} \times \mathbb{C P}^{1}$ be the image curve of bidegree $(d, d)$ obtained by application of the map $(\phi, \bar{\phi})$ to $\mathcal{C}^{\prime}$ and let $\widetilde{\mathcal{D}^{\prime}}$ be the normalization of $\mathcal{D}^{\prime}$. Let $\mu^{\prime}: \widetilde{\mathcal{D}^{\prime}} \rightarrow \mathcal{D}^{\prime}$ be the canonical birational map and, finally, let $\phi: \widetilde{\mathcal{D}^{\prime}} \rightarrow \mathbb{C P}$ be the composition of $\mu^{\prime}$ and the projection of $\mathbb{C P}^{1} \times \mathbb{C P}^{1}$ on its first factor. Then $\phi$ has degree $d$ and all the critical points of $\phi$ are real by Proposition 2.2 (3). Note that if $f$ is not conjugate to a real function by a Möbius transformation the same holds for $\phi$ as well. Hence, $\phi: \widetilde{D^{\prime}} \rightarrow \mathbb{C P}^{1}$ also violates the total reality conjecture. The image of $\widetilde{D^{\prime}} \stackrel{(\phi, \bar{\phi})}{\longrightarrow} \mathbb{C P}^{1} \times \mathbb{C P}^{1}$ coincides with $\mathcal{D}^{\prime}$, and the map $\mu^{\prime}: \widetilde{D^{\prime}} \rightarrow \mathcal{D}^{\prime}$ is birational. So $\mathcal{D}^{\prime}$ satisfies the assumptions i)-iii) of Proposition 1.5 for $g=g\left(\widetilde{D^{\prime}}\right)$ and $d=\delta>1$, see Proposition 2.2. Indeed, the map $\mathcal{C}^{\prime} \rightarrow \mathcal{D}^{\prime}$ lifts to a map $\mathcal{C}^{\prime} \rightarrow \widetilde{D^{\prime}}$ of degree $\delta=d^{\prime} / d$ with only simple ramifications whose number by the Riemann-Hurwitz formulas is $2 g\left(\mathcal{C}^{\prime}\right)-2-\delta\left(2 g\left(\widetilde{D^{\prime}}\right)-2\right)$. Hence the number of critical points of $f$ that are the preimages of cusps of $\mathcal{D}^{\prime}$ can be computed as $K=2 g\left(\mathcal{C}^{\prime}\right)-2+2 d^{\prime}-\left(2 g\left(\mathcal{C}^{\prime}\right)-2-\delta\left(2 g\left(\widetilde{D^{\prime}}\right)-2\right)\right)$. Note that each cusp has as preimages exactly $\delta$ critical points. Finally we compute the number of cusps of $\mathcal{D}^{\prime}$ as $\frac{1}{\delta} K=2 g\left(\widetilde{D^{\prime}}\right)-2+2 d$.

And conversely, exactly as in the above proof given a curve $\mathcal{D}^{\prime} \subset \mathbb{C P}^{1} \times \mathbb{C P}^{1}$ satisfying the assumptions i)-iii) of Proposition 1.5 we get a meromorphic function violating the total reality conjecture by composing the birational projection $\mu^{\prime}$ from the normalization $\widetilde{\mathcal{D}^{\prime}}$ to $\mathcal{D}^{\prime}$ with the projection of $\mathcal{D}^{\prime}$ on the first coordinate in $\mathbb{C P}^{1} \times \mathbb{C P}^{1}$

Now we are ready to prove Theorem 1.3 Indeed, if we assume that all the critical points of a generic meromorphic function $f:(\mathcal{C}, \sigma) \rightarrow \mathbb{C P}^{1}$ are real then $k$ in the above Proposition equals $2 d-2+2 g(\mathcal{C})$. Under the assumption $g(\mathcal{C})>\frac{d^{2}-4 d+3}{3}$ one gets $g(\mathcal{C})+k=2 d-2+3 g(\mathcal{C})>(d-1)^{2}$. Thus, the case when $\mathcal{C}$ maps birationally to $\mathcal{D}$ is impossible by Proposition [2.6. Since $d$ is prime the only other possible case is when the degree of the map $\mathcal{C} \rightarrow \mathcal{D}$ equals $d$ and therefore, the degree of the map $\mathcal{D} \rightarrow \mathbb{C P}^{1}$ equals 1 which by (2) of Proposition 2.2 gives that the total reality property holds.

Let us apply Theorem 1.3 to prove Corollary 1.4 .

Case $d=2$. Suppose that the degree $d$ of a meromorphic function $f:(\mathcal{C}, \sigma) \rightarrow \mathbb{C P}^{1}$ is equal to 2. That only leaves two possibilities: The first is that the map $\mathcal{C} \rightarrow \mathcal{D}$ has degree 2 and then by Proposition $2.2 f$ is real for some real structure $\left(\mathbb{C P}^{1}, \tau\right)$ on $\mathbb{C P}^{1}$. In particular, if the set $\mathcal{C}_{\sigma}$ of real points is nonempty then $\left(\mathbb{C P}^{1}, \tau\right)$ has the same property which means that it is equivalent to the standard real structure. The second is that the map $\mathcal{C} \rightarrow \mathcal{D}$ is birational and then by Proposition 2.6 we get 
$g(\mathcal{C})+k \leq 1^{2}=1$, where $k$ is the number of real critical points of $f$. In particular if $g(\mathcal{C})>0$ then there are no real critical points. Thus a hyper-elliptic map from a real curve $(\mathcal{C}, \sigma)$ is real if one of its critical points is real.

Case $d=3$. In this case again we have only two possibilities; either $f$ is real for a real structure on $\mathbb{C P}^{1}$ or $\mathcal{C} \rightarrow \mathcal{D}$ is birational in which case we have $g(\mathcal{C})+k \leq 2^{2}=4$. The case $g(\mathcal{C})=0$ was settled in 3 . Recall that the total number of critical points equals $2 d-2+2 g(\mathcal{C})$. But if $g(\mathcal{C})>0$ then $2 \cdot 3-2+3 g>4$ and this case of Theorem 1.4 is settled. Analogously to the case $d=2$ a function $f$ with the degree $d=3$ is real if it has more than $\max (4-g(\mathcal{C}), 1)$ real critical points.

Now we can finally start proving Theorem 1.7 Using a version of Proposition 2.2 we reduce the case of degree $d=4$ to the existence problem of a real curve on the ellipsoid Ell $=\left(\mathbb{C P}^{\mathbf{1}} \times \mathbb{C P}^{\mathbf{1}}, \mathbf{s}\right)$ of bi-degree $(4,4)$ with 8 ordinary real cusps and no other singularities. Indeed, arguing along the same lines as above we have three possibilities for the image $\mathcal{D}$ of $\mathcal{C}$ under the map $(f, \bar{f})$. Namely, $\mathcal{D}$ might have bi-degrees $(1,1),(2,2)$, or $(4,4)$. In the first case $f$ can be made real. In the second case, by Proposition 2.2 the projection on the first factor will give a map from the normalization $\widetilde{\mathcal{D}}$ of $\mathcal{D}$. The arithmetic genus $p_{a}(\mathcal{D})=1$, and the geometric genus $g(\widetilde{\mathcal{D}})$ of the normalization $\widetilde{D}$ does not exceed 1 . Let $\widetilde{h}: \mathcal{C} \rightarrow \widetilde{\mathcal{D}}$ be the lift of $h: \mathcal{C} \rightarrow$ $\mathcal{D}$. Note that if $p_{i} \in \mathcal{C}$ is a critical point of $f$ then either its image $h\left(p_{i}\right)$ is a cusp of $\mathcal{D}$ or $p_{i}$ is a ramification point of $\widetilde{h}$. The ramification divisor $R(\widetilde{h})=2 g(\mathcal{C})+2-4 g(\widetilde{\mathcal{D}})$. The number of cusps of $\mathcal{D}$ does not exceed 1 , whereas the number of distinct critical points of $f$ is $2 g(\mathcal{C})+6$. Note that any cusp has two critical points of $f$ as preimages. Therefore, we must have $\frac{1}{2}(2 g(\mathcal{C})+6-(2 g(\mathcal{C})+2-4 g(\widetilde{\mathcal{D}}))) \leq 1$ which is impossible.

We are hence left with the case when $\mathcal{D}$ has degree $(4,4)$. The only case when $2 \cdot 4-2+3 g(\mathcal{C}) \leq 9$ for $g(\mathcal{C})>0$ is the case of $g(\mathcal{C})=1$. If all the critical points $p_{1}, \ldots, p_{8}$ of $f: \mathcal{C} \rightarrow \mathbb{C P}^{1}$ are real, then we get a birational map $\mathcal{C}\left(p_{1}, \ldots, p_{8}\right) \rightarrow$ $\mathcal{D}$ and as then both $\mathcal{C}\left(p_{1}, \ldots, p_{8}\right)$ and $\mathcal{D}$ have arithmetic genus 9 , this map is an isomorphism. Hence $\mathcal{D}$ is a curve with 8 ordinary real cusps and no other singularities. To finish the proof of Theorem 1.7 we have to show that such curves do not exist. Since the proof of this claim requires a lot of additional notation and techniques we decided to place it into a separate section.

\section{INTERSECTION LATTICES}

We will need a number of standard notions from the lattice theory and $K 3$ surfaces.

3.1. Discriminant forms. A lattice is a finitely generated free abelian group $L$ supplied with a symmetric bilinear form $b: L \otimes L \rightarrow \mathbb{Z}$. We abbreviate $b(x, y)=x \cdot y$ and $b(x, x)=x^{2}$. A lattice $L$ is even if $x^{2}=0 \bmod 2$ for all $x \in L$. As the transition matrix between two integral bases has determinant \pm 1 , the determinant $\operatorname{det} L \in \mathbb{Z}$ (i.e., the determinant of the Gram matrix of $b$ in any basis of $L$ ) is well defined. A lattice $L$ is called nondegenerate if the determinant $\operatorname{det} L \neq 0$; it is called unimodular if $\operatorname{det} L= \pm 1$.

Given a lattice $L$, the bilinear form can be extended to $L \otimes \mathbb{Q}$ by linearity. If $L$ is nondegenerate, the dual group $L^{\vee}=\operatorname{Hom}(L, \mathbb{Z})$ can be identified with the subgroup

$$
\{x \in L \otimes \mathbb{Q} \mid x \cdot y \in \mathbb{Z} \text { for all } x \in L\} .
$$


In particular, $L \subset L^{\vee}$. The quotient $L^{\vee} / L$ is a finite group; it is called the discriminant group of $L$ and is denoted by $\operatorname{discr} L$ or $\mathcal{L}$. The discriminant group $\mathcal{L}$ inherits from $L \otimes \mathbb{Q}$ a symmetric bilinear form $\mathcal{L} \otimes \mathcal{L} \rightarrow \mathbb{Q} / \mathbb{Z}$, called the discriminant form, and, if $L$ is even, its quadratic extension $\mathcal{L} \rightarrow \mathbb{Q} / 2 \mathbb{Z}$. When speaking about the discriminant groups, their (anti-)isomorphisms, etc., we always assume that the discriminant form (and its quadratic extension if the lattice is even) is taken into account. One has $\# \mathcal{L}=|\operatorname{det} L|$; in particular, $\mathcal{L}=0$ if and only if $L$ is unimodular.

In what follows we denote by $\mathbf{U}$ the hyperbolic plane, i.e., the lattice generated by a pair of vectors $u, v$ (referred to as a standard basis for $\mathbf{U}$ ) with $u^{2}=v^{2}=0$ and $u \cdot v=1$. Furthermore, given a lattice $L$, we denote by $n L, n \in \mathbb{N}$, the orthogonal sum of $n$ copies of $L$, and by $L(p), p \in \mathbb{Q}$, the lattice obtained from $L$ by multiplying the form by $q$ (assuming that the result is still an integral lattice). The notation $n \mathcal{L}$ is also used for the orthogonal sum of $n$ copies of a discriminant group $\mathcal{L}$.

Two lattices $L_{1}, L_{2}$ are said to have the same genus if all localizations $L_{i} \otimes \mathbb{Q}_{p}$, $p$ prime, and $L_{i} \otimes \mathbb{Q}$ are pairwise isomorphic. As a general rule, it is relatively easy to compare the genera of two lattices; for example, the genus of an even lattice is determined by its signature and the isomorphism class of the discriminant group, see 9 . In the same paper [9] one can find a few classes of lattices whose genus is known to contain a single isomorphism class.

Following V. V. Nikulin, we denote by $\ell(\mathcal{L})$ the minimal number of generators of a finite group $\mathcal{L}$ and, for a prime $p$, let $\ell_{p}(\mathcal{L})=\ell\left(\mathcal{L} \otimes \mathbb{Z}_{p}\right)$. (Here $\mathbb{Z}_{p}$ stands for the cyclic group $\mathbb{Z} / p \mathbb{Z}$.) If $L$ is a nondegenerate lattice, there is a canonical epimorphism $\operatorname{Hom}\left(L, \mathbb{Z}_{p}\right) \rightarrow \mathcal{L} \otimes \mathbb{Z}_{p}$. It is an isomorphism if and only if $\operatorname{rank} L=\ell_{p}(\mathcal{L})$.

An extension of a lattice $L$ is another lattice $M$ containing $L$. An extension is called primitive if $M / L$ is torsion free. In what follows we are only interested in the case when both $L$ and $M$ are even. The relation between extensions of even lattices and there discriminant forms was studied in details by Nikulin; next two theorems are found in 9 .

Theorem 3.1. Given a nondegenerate even lattice $L$, there is a canonical one-toone correspondence between the set of isomorphism classes of finite index extensions $M \supset L$ and the set of isotropic subgroups $\mathcal{K} \subset \mathcal{L}$. Under this correspondence one has $M=\left\{x \in L^{\vee} \mid x \bmod L \in \mathcal{K}\right\}$ and $\operatorname{discr} M=\mathcal{K}^{\perp} / \mathcal{K}$.

Theorem 3.2. Let $M \supset L$ be a primitive extension of a nondegenerate even lattice $L$ to a unimodular even lattice $M$. Then there is a canonical anti-isometry $\mathcal{L} \rightarrow$ discr $L^{\perp}$ of discriminant forms; its graph is the kernel $\mathcal{K} \subset \mathcal{L} \oplus$ discr $L^{\perp}$ of the finite index extension $M \supset L \oplus L^{\perp}$, see Theorem 3.1. Furthermore, a pair of autoisometries of $L$ and $L^{\perp}$ extends to an auto-isometry of $M$ if and only if the induced automorphisms of $\mathcal{L}$ and discr $L^{\perp}$, respectively, agree via the above anti-isometry of the discriminant groups.

The general case $M \supset L$ splits into the finite index extension $\tilde{L} \supset L$ and primitive extension $M \supset \tilde{L}$, where

$$
\tilde{L}=\{x \in M \mid n x \in L \text { for some } n \in \mathbb{Z}\}
$$

is the primitive hull of $L$ in $M$.

A root in an even lattice $L$ is a vector $r \in L$ of square -2 . A root system is an even negative definite lattice generated by its roots. Recall that each root system splits (uniquely up to order of the summands) into orthogonal sum of indecomposable 
root systems, the latter being those of types $\mathbf{A}_{p}, p \geq 1, \mathbf{D}_{q}, q \geq 4, \mathbf{E}_{6}, \mathbf{E}_{7}$, or $\mathbf{E}_{8}$, see [2]. A finite index extension $\Sigma \subset \tilde{\Sigma}$ of a root system $\Sigma$ is called quasi-primitive if each root of $\tilde{\Sigma}$ belongs to $\Sigma$.

Each root system that can be embedded in $\mathbf{E}_{8}$ is unique in its genus, see 9]. In what follows we need the discriminant group $\operatorname{discr} \mathbf{A}_{2}=\left\langle-\frac{2}{3}\right\rangle$ : it is the cyclic group $\mathbb{Z}_{3}$ generated by an element of square $-\frac{2}{3} \bmod 2 \mathbb{Z}$.

3.2. $K 3$-surfaces and ramified double coverings of $\mathbb{C P}^{1} \times \mathbb{C P}^{1}$. A $K 3$-surface is a nonsingular compact connected and simply connected complex surface with trivial first Chern class. From the Castelnuovo-Enriques classification of surfaces it follows that all $K 3$-surfaces form a single deformation family. In particular, they are all diffeomorphic, and the calculation for an example (say, a quartic in $\mathbb{C P}^{3}$ ) shows that

$$
\chi(X)=24, \quad h^{2,0}(X)=1, \quad h^{1,1}(X)=20 .
$$

(see, for instance, [1]). Hence, the intersection lattice $H_{2}(X ; \mathbb{Z})$ is an even (since $w_{2}(X)=K_{X} \bmod 2=0$ ) unimodular (as intersection lattice of any closed 4manifold) lattice of rank 22 and signature -16 . All such lattices are isomorphic to $L=2 \mathbf{E}_{8} \oplus 3 \mathbf{U}$. In particular, the quadratic space $H_{2}(X ; \mathbb{R}) \cong L \otimes \mathbb{R}$ has three positive squares; for a maximal positive definite subspace one can choose the subspace spanned by the real and imaginary parts of the class $[\omega]$ of a holomorphic form $\omega$ on $X$ and the class $[\rho]$ of the fundamental form of a Kähler metric on $X$. (We identify the homology and cohomology via the Poincaré duality.)

A real $K 3$-surface is a pair ( $X$, conj), where $X$ is a $K 3$-surface and conj : $X \rightarrow X$ an anti-holomorphic involution., i.e., a real structure on $X$. The $(+1)$-eigenlattice $\operatorname{ker}\left(1-\operatorname{conj}_{*}\right) \subset H_{2}(X ; \mathbb{Z})$ of $\operatorname{conj}_{*}$ is hyperbolic, i.e., it has one positive square in the diagonal form over $\mathbb{R}$. This follows, e.g., from the fact that $\omega$ and $\rho$ above can be chosen so that $\operatorname{conj}_{*}[\omega]=[\bar{\omega}]$ and $\operatorname{conj}_{*}[\rho]=-[\rho]$.

Let $Y=\mathbb{C P}^{1} \times \mathbb{C P}^{1}$ and let $C \subset Y$ be an irreducible curve of bi-degree $(4,4)$ with at worst simple singularities (i.e., those of type $\mathbf{A}_{p}, \mathbf{D}_{q}, \mathbf{E}_{6}, \mathbf{E}_{7}$, or $\mathbf{E}_{8}$ ). Then, the minimal resolution $X$ of the double covering of $Y$ ramified along $C$ is a $K 3$ surface. Recall that the standard ellipsoid is the pair Ell $=(Y, s)$ where $s$ is the anti-holomorphic involution $s: Y \rightarrow Y, s:(x, y) \mapsto(\bar{y}, \bar{x})$. If $C$ is $s$-invariant, the involution $s$ lifts to two different real structures on $X$, which commute with each other and with the deck translation of the covering $X \rightarrow Y$. Choose one of the two lifts and denote it by conj.

Let $l_{1}, l_{2} \in H_{2}(X ; \mathbb{Z})$ be the pull-backs of the classes of two lines belonging to the two rulings of $Y$. Then $l_{1}^{2}=l_{2}^{2}=0$ and $l_{1} \cdot l_{2}=2$, i.e., $l_{1}$ and $l_{2}$ span a sublattice $\mathbf{U}(2)$, and $\operatorname{conj}_{*}$ acts via

$$
l_{1} \mapsto-l_{2}, \quad l_{2} \mapsto-l_{1} .
$$

Each (simple) singular point of $C$ gives rise to a singular point of the double covering, and the exceptional divisors of its resolution span a root system in $H_{2}(X ; \mathbb{Z})$ of the same type ( $\mathbf{A}, \mathbf{D}$, or $\mathbf{E})$ as the original singular point. These root systems are orthogonal to each other and to $l_{1}, l_{2}$; denote their sum by $\Sigma$. If all singular points are real, then $\operatorname{conj}_{*}$ acts on $\Sigma$ via multiplication by $(-1)$.

Lemma 3.3. The sublattice $\Sigma \subset H_{2}(X ; \mathbb{Z})$ is quasi-primitive in its primitive hull.

Proof. Let $r \notin \Sigma$ be a root in the primitive hull of $\Sigma$. Since, obviously, $\Sigma \subset \operatorname{Pic} X$ and $H_{2}(X ; \mathbb{Z}) / \mathrm{Pic} X$ is torsion free, one has $r \in \operatorname{Pic} X$. Then, the Riemann-Roch 
theorem implies that either $r$ or $-r$ is effective, i.e., it is realized by a $(-2)$-curve in $X$ (possibly, reducible), which is not contracted by the blow down (as $r \notin \Sigma$ ). On the other hand, $r$ is orthogonal to $l_{1}$ and $l_{2}$. Hence, the curve projects to a curve in $Y$ orthogonal to both the rulings, which is impossible.

\subsection{The calculation.}

Lemma 3.4. The lattice $\Sigma=3 \mathbf{A}_{2}$ has no non-trivial quasi-primitive extensions.

Proof. Up to automorphism of $3 \mathbf{A}_{2}$, the discriminant group discr $3 \mathbf{A}_{2} \cong 3\left\langle-\frac{2}{3}\right\rangle$ has a unique isotropic element, which is the sum of all three generators. Then, for the corresponding extension $\tilde{\Sigma} \supset \Sigma$ one has discr $\tilde{\Sigma}=\left\langle\frac{2}{3}\right\rangle$, i.e., $\tilde{\Sigma}$ has the genus of $\mathbf{E}_{6}$. Since the latter is unique in its genus (see 9 ), one has $\tilde{\Sigma} \cong \mathbf{E}_{6}$. Alternatively, one can argue that, on one hand, an imprimitive extension of $3 \mathbf{A}_{2}$ is unique and, on the other hand, an embedding $3 \mathbf{A}_{2} \subset \mathbf{E}_{6}$ is known: if $2 \mathbf{A}_{2}$ is embedded into $\mathbf{E}_{6}$ via the Dynkin diagrams, the orthogonal complement is again a copy of $\mathbf{A}_{2}$.

Lemma 3.5. Up to automorphism, the lattice $\Sigma=8 \mathbf{A}_{2}$ has two non-trivial quasiprimitive extensions $\tilde{\Sigma} \supset \Sigma$; one has $\ell_{3}(\tilde{\Sigma})=6$ or 4 .

Proof. We will show that there are at most two classes. The fact that the two extensions constructed are indeed quasi-primitive is rather straightforward, but it is not needed in the sequel.

Let $\mathcal{S}=\operatorname{discr} \Sigma \cong 8\left\langle-\frac{2}{3}\right\rangle$ be the discriminant group, and let $G$ be the set of generators of $\mathcal{S}$. The automorphisms of $\Sigma$ act via transpositions of $G$ or reversing some of the generators. (Recall that the decomposition of a definite lattice into an orthogonal sum of indecomposable summands is unique up to transposing the summands.) For an element $a \in \mathcal{S}$ define its support $\operatorname{supp} a \subset G$ as the subset consisting of the generators appearing in the expansion of $a$ with a non-zero coefficient. Since each nontrivial summand in the expansion of an element $a \in \mathcal{S}$ contributes $-\frac{2}{3} \bmod 2 \mathbb{Z}$ to the square, $a$ is isotropic if and only if \# $\operatorname{supp} a=0 \bmod 3$; in view of Lemma 3.4 such an element cannot belong to the kernel of a quasi-primitive extension unless $\# \operatorname{supp} a=6$. (Indeed, if $\# \operatorname{supp} a=3$, then $a$ belongs to the discriminant group of the sum $\Sigma^{\prime}$ of certain three of the eight $\mathbf{A}_{2}$-summands of $\Sigma$, and already $\Sigma^{\prime}$ is not primitive, hence, not quasi-primitive.)

All elements $a \in \mathcal{S}$ with \# $\operatorname{supp} a=6$ form a single orbit of the action of $\operatorname{Aut} \Sigma$, thus giving rise to a unique isomorphism class of quasi-primitive extensions $\tilde{\Sigma} \supset \Sigma$ with $\ell_{3}(\operatorname{discr} \tilde{\Sigma})=6$. Consider the extensions with $\ell_{3}(\operatorname{discr} \tilde{\Sigma})=4$, i.e., those whose kernel $\mathcal{K}$ is isomorphic to $\mathbb{Z}_{3} \oplus \mathbb{Z}_{3}$. Up to the action of Aut $\Sigma$ the generators $g_{1}, \ldots, g_{8}$ of $\mathcal{S}$ and two elements $a_{1}, a_{2}$ generating $\mathcal{K}$ can be chosen so that $a_{1}=g_{1}+\ldots+g_{6}$ and $a_{2}=\left(g_{1}+\ldots+g_{p}-g_{p+1}-\ldots-g_{p+q}\right)+\sigma$, where $\sigma=0, g_{7}$, or $g_{7}+g_{8}$ and $p \geq q \geq 0$ are certain integers such that $p+q=\#\left(\operatorname{supp} a_{1} \cap \operatorname{supp} a_{2}\right) \leq 6$. Since $\operatorname{supp} a_{1}$ and $\operatorname{supp} a_{2}$ are two six element sets and \#( $\left.\operatorname{supp} a_{1} \cup \operatorname{supp} a_{2}\right) \leq 8$, one has $p+q \geq 4$. Furthermore, since $a_{1} \cdot a_{2}=\frac{2}{3}(p-q) \bmod \mathbb{Z}=0$, one has $p-q=0 \bmod 3$. This leaves three pairs of values: $(p, q)=(2,2),(3,3)$, or $(4,1)$. In the first case, $(p, q)=(2,2)$, one does obtain a quasi-primitive extension, unique up to automorphism. In the other two cases one has \# $\operatorname{supp}\left(a_{1}-a_{2}\right)=3$ and, hence, the extension is not quasi-primitive due to Lemma 3.4 (cf. the previous paragraph).

Note that, in the only quasi-primitive case $(p, q)=(2,2)$, for any pair $a_{1}, a_{2}$ of generators of $\mathcal{K}$ one has

$$
\operatorname{supp} a_{1} \cup \operatorname{supp} a_{2}=G \quad \text { and } \quad \#\left(\operatorname{supp} a_{1} \cap \operatorname{supp} a_{2}\right)=4 .
$$


As a by-product, the same relations must hold for any two independent (over $\mathbb{Z}_{3}$ ) elements $a_{1}, a_{2}$ in the kernel of any quasi-primitive extension.

Now, assume that the kernel of the extension $\tilde{\Sigma} \supset \Sigma$ contains $\mathbb{Z}_{3} \oplus \mathbb{Z}_{3} \oplus \mathbb{Z}_{3}$, i.e., $\ell_{3}(\operatorname{discr} \tilde{\Sigma})<4$. Pick three independent (over $\mathbb{Z}_{3}$ ) elements $a_{1}, a_{2}, a_{3}$ in the kernel. In view of (3.1), the principle of inclusion and exclusion implies that \# $\left(\operatorname{supp} a_{1} \cap \operatorname{supp} a_{2} \cap \operatorname{supp} a_{3}\right)=2$. Important is the fact that the intersection is nonempty. Hence, with appropriate choice of the signs, there is a generator of $\mathcal{S}$, say, $g_{1}$, whose coefficients in the expansions of all three elements $a_{i}$ coincide. Then the two differences $b_{1}=a_{1}-a_{3}$ and $b_{2}=a_{2}-a_{3}$ belong to the kernel, are independent, and their supports do not contain $g_{1}$. This contradicts to (3.1).

Proposition 3.6. Let $L$ be a lattice isomorphic to $2 \mathbf{E}_{8} \oplus 3 \mathbf{U}$, and let $S=\Sigma \oplus \mathbf{U}(2)$ be a sublattice of $L$ with $\Sigma \cong 8 \mathbf{A}_{2}$ quasi-primitive in its primitive hull. Then $L$ has no involutive automorphism $c$ acting identically on $\Sigma$, interchanging the two elements of a standard basis of $\mathbf{U}(2)$, and having exactly two positive squares in the $(+1)$-eigenlattice $L^{+c}=\operatorname{ker}(1-c) \subset L$.

Proof. Assume that such an involution $c$ exists. Let $\tilde{\Sigma}$ and $\tilde{S}$ be the primitive hulls of $\Sigma$ and $S$, respectively, in $L$, and let $T=S^{\perp}$ be the orthogonal complement. The lattice $T$ has rank 4 and signature 0 , i.e., it has two positive and two negative squares.

Since discr $\mathbf{U}(2)=\mathbb{Z}_{2} \oplus \mathbb{Z}_{2}$ (as a group) has 2-torsion only, the 3 -torsion parts $(\operatorname{discr} \tilde{\Sigma}) \otimes \mathbb{Z}_{3}$ and $(\operatorname{discr} \tilde{S}) \otimes \mathbb{Z}_{3}$ coincide. In particular, $c$ must act identically on $(\operatorname{discr} \tilde{S}) \otimes \mathbb{Z}_{3}$ (as, by the assumption, so it does on $\Sigma$ ) and, hence, on $(\operatorname{discr} T) \otimes$ $\mathbb{Z}_{3}$, see Theorem [3.2 Furthermore, due to Lemma [3.5] one has $\ell_{3}(\operatorname{discr} T)=$ $\ell_{3}(\operatorname{discr} \tilde{S}) \geq 4$. On the other hand, $\ell_{3}(\operatorname{discr} T) \leq \operatorname{rank} T=4$. Hence, $\ell_{3}(\operatorname{discr} T)=$ $\operatorname{rank} T=4$ and the canonical homomorphism $T^{\vee} \otimes \mathbb{Z}_{3} \rightarrow(\operatorname{discr} T) \otimes \mathbb{Z}_{3}$ is an isomorphism. Thus, $c$ must also act identically on $T^{\vee} \otimes \mathbb{Z}_{3}$ and, hence, both on $T^{\vee}$ and $T \subset T^{\vee}$. Indeed, for any free abelian group $V$, any involution $c: V \rightarrow V$, and any odd prime $p$, one has a direct sum decomposition $V \otimes \mathbb{Z}_{p}=\left(V^{+c} \otimes \mathbb{Z}_{p}\right) \oplus\left(V^{-c} \otimes \mathbb{Z}_{p}\right)$. Hence, $c$ acts identically on $V$ (i.e., $V^{-c}=0$ ) if and only if it acts identically on $V \otimes \mathbb{Z}_{p}$ (i.e., $V^{-c} \otimes \mathbb{Z}_{p}=0$ ).

It remains to notice that, under the assumptions, the skew-invariant part $S^{-c}=$ $\operatorname{ker}(1+c) \cong \Sigma \oplus\langle-4\rangle$ is negative definite. Since the total skew-invariant part $L^{-c}$ has exactly one $(=3-2)$ positive square, one of the two positive squares of $T$, should fall to $T^{-c}$ and the other, to $T^{+c}$ In particular, $T^{-c} \neq 0$, and the action of $c$ on $T$ is not identical.

Now we have finally reached the goal of this section.

Theorem 3.7. The ellipsoid $\mathbf{E l l}=(Y, s)$ (see 1 and 3 3.3) does not contain a real curve $C$ of bi-degree $(4,4)$ having eight real cusps (and no other singularities).

Proof. Any such curve $C$ would be irreducible; hence, as in 3.3 it would give rise to a sublattice $8 \mathbf{A}_{2} \oplus \mathbf{U}(2) \subset L=H_{2}(X ; \mathbb{Z}) \cong 2 \mathbf{E}_{8} \oplus 3 \mathbf{U}$ and involution $c=-$ conj $_{*}: L \rightarrow L$ which do not exist due to Proposition 3.6

\section{REMARKS AND PROBLEMS}

I. Analogously to the total reality property for rational curves one can ask a similar question for projective curves of any genus, namely 
Problem 4.1. Given a real algebraic curve $(\mathcal{C}, \sigma)$ with compact $\mathcal{C}$ and nonempty real part $\mathcal{C}_{\sigma}$ and a complex algebraic map $\Psi: \mathcal{C} \rightarrow \mathbb{C P}^{n}$ such that the inverse images of all the flattening points of $\Psi(\mathcal{C})$ lie on the real part $\mathcal{C}_{\sigma} \subset \mathcal{C}$ is it true that $\Psi$ is a real algebraic up to a projective automorphism of the image $\mathbb{C P}^{n}$ ?

The feeling is that this problem has a negative answer.

II. In the recent 4 the authors found another generalization of the conjecture on total reality in case of the usual rational functions.

Problem 4.2. Extend the results of [4] to the case of meromorphic functions on curves of higher genera.

\section{REFERENCES}

[1] W. Barth, C. Peters, A. Van de Ven, Compact Complex Surfaces. Springer-Verlag. 1984.

[2] N. Bourbaki, Éléments de mathématique. Fasc. XXXIV. Groupes et algèbres de Lie. Chap. 4-6, Actualités Scientifiques et Industrielles vol 1337, Hermann, Paris, 1968.

[3] A. Eremenko, A. Gabrielov, Rational functions with real critical points and the B. and M. Shapiro conjecture in real enumerative geometry. Ann. of Math. (2) 155 (2002), no. 1, 105129.

[4] A. Eremenko, A. Gabrielov, M. Shapiro, A. Vainshtein, Rational functions and real Schubert calculus, math.AG/0407408

[5] T. Ekedahl, B. Shapiro, M. Shapiro, First steps towards total reality of meromorphic functions, submitted to Moscow Mathematical Journal.

[6] D. Gudkov, E. Shustin, Classification of nonsingular eighth-order curves on an ellipsoid. (Russian) Methods of the qualitative theory of differential equations, (1980), 104-107, Gor'kov. Gos. Univ., Gorki.

[7] V. Kharlamov and F. Sottile, Maximally inflected real rational curves, Mosc. Math. J. 3 (2003), no. 3, 947-987, 1199-1200.

[8] E. Mukhin, V. Tarasov, A. Varchenko, The B. and M. Shapiro conjecture in real algebraic geometry and the Bethe ansatz, preprint math.AG/0512299

[9] V. V. Nikulin, Integer quadratic forms and some of their geometrical applications, Izv. Akad. Nauk SSSR, Ser. Mat vol. 43, (1979), no. 1, 111-177; English transl. in Math. USSR-Izv. vol 43 (1979), 103-167.

[10] J. Ruffo, Y. Sivan, E. Soprunova, and F. Sottile, Experimentation and conjectures in the real Schubert calculus for flag manifolds, Preprint (2005), math.AG/0507377

[11] F. Sottile, Enumerative geometry for the real Grassmannian of lines in projective space, Duke Math J., 87 (1997), 59-85.

[12] F. Sottile, Enumerative geometry for real varieties, Proc. of Symp. Pur. Math., 62 (1997), no. $1,435-447$.

[13] F. Sottile, Real Schubert calculus: polynomial systems and a conjecture of Shapiro and Shapiro, Experiment. Math., 9, (2000), no. 2, 161-182.

[14] F. Sottile, The special Schubert calculus is real, Electronic Res. Ann. of the AMS, 5 (1999), no. $1,35-39$.

[15] F. Sottile, website - www.expmath.org/extra/9.2/sottile.

[16] J. Verschelde, Numerical evidence for a conjecture in real algebraic geometry, Experiment. Math. 9 (2000), no. 2, 183-196.

[17] R. J. Walker, Algebraic Curves. Princeton Mathematical Series, vol. 13. Princeton University Press, Princeton, N. J., 1950. $\mathrm{x}+201$ pp. 
Department of Mathematics, Bilkent University, Bilkent, Ankara 06533, Turkey

E-mail address: degt@fen.bilkent.edu.tr

Department of Mathematics, Stockholm University, SE-106 91 Stockholm, Sweden

E-mail address: teke@math.su.se

IRMA, Université Louis Pasteur, 7 rue René Descartes, 67084 Strasbourg Cedex, FRANCE

E-mail address: itenberg@math.u-strasbg.fr

Department of Mathematics, Stockholm University, SE-106 91 Stockholm, Sweden

E-mail address: shapiro@math.su.se

Department of Mathematics, Michigan State University, East Lansing, Mi 488241027, USA

E-mail address: mshapiro@math.msu.edu 